\title{
Journal of Cancer
}

Review

$2011 ; 2: 193-199$

\section{Thyroid Cancer: Burden of Illness and Management of Disease}

\author{
Rebecca L. Brown ${ }^{凶}$, Jonas A. de Souza, Ezra EW Cohen \\ Department of Medicine, The University of Chicago, Chicago, IL 60637, USA
}

$\triangle$ Corresponding author: Rebecca L. Brown, MD, Assistant Professor of Medicine, The University of Chicago, 5841 S. Maryland Avenue, Chicago, IL 60637. Tel: 773-702-9458; Fax: 773-834-0486; rbrown3@medicine.bsd.uchicago.edu

(C) Ivyspring International Publisher. This is an open-access article distributed under the terms of the Creative Commons License (http://creativecommons.org/ licenses/by-nc-nd/3.0/). Reproduction is permitted for personal, noncommercial use, provided that the article is in whole, unmodified, and properly cited.

Received: 2011.02.03; Accepted: 2011.03.24; Published: 2011.04.04

\begin{abstract}
Objective: The incidence of thyroid cancer, the most common endocrine malignancy, has increased dramatically in the last fifty years. This article will review the standard approach to thyroid cancer treatment as well as novel therapies under investigation. We will also address potential cost considerations in the management of thyroid cancer.

Study Design: A comprehensive literature search was performed.

Methods: Review article.

Results: The high prevalence of thyroid cancer and the availability of novel therapies for patients with metastatic disease have potential economic implications that have not been well-studied. Because many patients likely have very low morbidity from their cancers, better tools to identify the lowest risk patients are needed in order to prevent overtreatment. Improved risk stratification should include recognizing patients who are unlikely to benefit from radioactive iodine therapy after initial surgery and identifying those with indolent and asymptomatic metastatic disease that are unlikely to benefit from novel therapies. In patients with advanced incurable disease, randomized-controlled studies to assess the efficacy of novel agents are needed to determine if the costs associated with new agents are warranted.

Conclusions: Health care costs associated with the increased diagnosis of thyroid cancer remain unknown but are worthy of further research.
\end{abstract}

Key words: differentiated thyroid cancer, radioactive iodine, targeted therapy, clinical trials, pharmacoeconomics

\section{Background/Epidemiology of Thyroid Can- cer in the United States}

Thyroid cancer is the most common endocrine malignancy, with an estimated 44,670 new cases diagnosed in the United States in 2010. Its prevalence continues to rise; in 2008 it became the sixth most diagnosed cancer in women. ${ }^{1}$ For reasons that are unclear, thyroid cancer is 2-3 fold more common in females than males. Although the peak incidence of thyroid cancer diagnosis is 45 to 49 years in women and 65 to 69 years in men, it does affect young people. Thyroid cancer accounts for approximately $10 \%$ of malignancies diagnosed in persons aged 15 to 29 years. $^{2}$

Thyroid carcinoma can arise from either follicular or non-follicular thyroid cells. Follicular cancers include papillary thyroid cancer (PTC, 80\%), follicular thyroid cancer (FTC, up to $11 \%$ ), Hürthle cell cancer (HCC, 3\%) and anaplastic thyroid cancer (ATC, 2\%). PTC and FTC, which together account for the vast majority of cancers, are commonly referred to together as differentiated thyroid cancer (DTC). HCC, a subtype of FTC, is often classified on its own because it has a distinct histological appearance and is often less responsive to standard therapy. Similar to HCC, 
several subtypes of DTC, including tall cell, columnar and insular thyroid cancers, are thought to be more aggressive. In contrast to DTC, ATC represents undifferentiated thyroid cancer. Medullary thyroid cancer (MTC) arises from non-follicular thyroid cells called calcitonin-producing cells and accounts for about $4 \%$ of thyroid cancers.

PTC accounts for the increase in new diagnoses of thyroid carcinoma. The reason for the increase in incident and prevalent cases is not clear, but many believe it is due to the widespread use of radiology tests done for other reasons that detect small nonpalpable thyroid cancers. Consistent with this theory is that the death rate from thyroid cancer has remained stable despite the increase in cases. However, a recent analysis suggests that all stages of thyroid cancer are increasing, a finding that cannot be explained by surveillance alone. ${ }^{3}$ Because PTC accounts for the increase in thyroid cancer burden, this review will focus on its management. Since PTC and FTC are often treated with similar therapy, we will collectively refer to this group of cancers as DTC.

DTC is considered the least aggressive type of thyroid cancer and has, in general, an excellent prognosis. Overall, greater than $90 \%$ of patients with thyroid cancer are alive at 10 years after diagnosis. This reassuring number is attributed to the fact that most people with thyroid cancer have low-risk disease. However, a small percentage of patients with DTC exhibit more aggressive disease. Of the approximately 1,690 people who die yearly from thyroid cancer in the United States, about $70 \%$ percent carry the diagnosis of DTC. ${ }^{1,} 4$ The prognosis of patients with metastatic DTC is difficult to predict. Some patients with metastatic DTC have stable or slow-growing disease and maintain good quality of life without therapy. Others, however, develop progressive disease that is not amenable to traditional therapy and, as a result, suffer increased morbidity and mortality from their cancer. Five-year survival is lower in patients with distant disease $(56 \%)$ compared with local $(99.7 \%)$ or regional $(96.9 \%)$ disease. ${ }^{1}$ Although it is difficult to predict who will go on to develop progressive disease, certain factors are implicated in increasing an individual's particular risk for developing progressive cancer. For example, age greater than 45 years of age, male gender, radioactive iodine resistance, and positive FDG uptake on PET scanning have been associated with poorer prognosis in DTC.

\section{Current Treatment Options in Thyroid Can- cer}

Initial treatment for DTC includes surgery, ra- dioactive iodine treatment and thyroid hormone suppression therapy. Standard of care recommendations are based largely on retrospective data. A prospective randomized trial to determine optimal therapy would be prohibitively long and expensive because survival rates are excellent. The lack of prospective data may lead to overtreatment of patients with low-risk disease who may have had no morbidity or mortality from their cancers if left untreated. Clinically unrecognized thyroid cancer has been seen in $4-35.6 \%$ of autopsies, which is far greater than prevalence of diagnosed cancer. ${ }^{5-8}$ Still, because up to $35 \%$ of patients, including those with low-risk tumors, have recurrences, ${ }^{9}$ most clinicians recommend therapy.

Total thyroidectomy is the preferred surgery for DTC. Lobectomy is only considered appropriate for small, isolated tumors (less than $1 \mathrm{~cm}$ ) without evidence of local spread. In retrospective studies, total thyroidectomy has been shown to improve disease-free survival and reduce recurrence rates. ${ }^{10-12}$ Total thyroidectomy allows for adequate staging and, when clinically appropriate, subsequent therapy with radioactive iodine (RAI, $\left.{ }^{131} \mathrm{I}\right)$. In addition, as many as $30-85 \%$ of patients have multifocal disease; ${ }^{13}$ this is not always appreciated until surgical specimens are evaluated.

A small amount of thyroid tissue, called the thyroid remnant, is often left after total thyroidectomy. RAI can be administered postoperatively to destroy any remaining thyroid cells, normal or malignant. RAI improves the specificity of future surveillance imaging to detect recurrent disease. It also allows clinicians to monitor serum thyroglobulin, a protein made by thyroid follicular cells, as a marker of disease. Because thyroid stimulating hormone (TSH) stimulation increases iodine uptake in thyroid cells, patients are withdrawn from thyroid hormone therapy or treated with recombinant human TSH (rhTSH) in order to drive ${ }^{131}$ I into remaining cells. Most commonly, therapy with ${ }^{131}$ I is given as fixed doses, with small cancers confined to the thyroid receiving low doses $(30 \mathrm{mCi})$ and more advanced cancers receiving higher doses (usually up to $200 \mathrm{mCi}$ when lung metastases are present).

Data suggest that high-risk patients (Stage III or IV) gain from RAI in terms of decreased disease progression and mortality. ${ }^{13-15}$ In contrast, the benefits of RAI in low-risk patients are uncertain and should be used selectively. Data suggest that RAI decreases recurrence rates in low-risk patients with tumors greater than $1.5 \mathrm{~cm}$, but whether it improves cause-specific survival is unclear. ${ }^{12,14-16}$ RAI treatment has not been shown to decrease recurrence rates or death in pa- 
tients with isolated tumors less than $1.5 \mathrm{~cm} .{ }^{12,17,18}$ Hay et al., reviewed PTC cases managed at the Mayo Clinic between 1940 - 1999. ${ }^{18}$ Despite the increased use of RAI during this period, no significant improvement in cause-specific mortality or tumor recurrence were observed in low-risk patients. These findings suggest that RAI should not be given routinely to low-risk patients. Other researchers have suggested basing RAI decision-making in low-risk patients on whether a stimulated $\mathrm{Tg}$ is detectable three months postoperatively. ${ }^{19}$ Vaisman et al. found that of 104 patients, $56.7 \%$ had an undetectable stimulated $\mathrm{Tg}$; only 1 of these patients received RAI. If widely applied, less RAI administration may decrease health care spending. In addition to cost, RAI does have potential side effects, including damage to salivary glands, bone marrow, and gonads, especially when given in high and cumulative doses. Therefore, its use should be targeted at patients who are likely benefit from therapy.

The third part to standard DTC therapy is thyroid hormone suppression therapy. Because TSH stimulates thyroid growth, the goal of suppression therapy is to keep TSH levels low. The degree of TSH suppression depends on the risk of the cancer, with only high-risk cancers requiring aggressive lowering of TSH. Several series have shown decreased recurrence rates and cancer-related mortality with thyroid hormone suppression therapy in high-risk patients. ${ }^{20}$ There is no evidence that low risk patients require suppression. ${ }^{13}$ In fact, the increased risk of atrial fibrillation and bone loss from TSH suppression make aggressive TSH-lowering undesirable in low-risk patients.

Because recurrences have been reported sometimes decades from initial treatment, ${ }^{9}$ patients with thyroid cancer require lifelong monitoring. Surveillance tests include measuring Tg levels while on and off thyroid hormone (or stimulated with rhTSH), neck ultrasounds, and 131I total body scans (TBSs). For patients with detectable Tg and negative TBSs, FDG-PET scanning is commonly employed. Which tests should be done and the time interval between testing must be tailored to the individual recurrence risk of the patient.

\section{Therapeutic Options for Advanced DTC}

Patients with progressive DTCs that are not responsive to standard treatment require additional therapy. Treatment should focus both on gaining local disease control in the neck as well as the management of systemic disease. Neck dissection should be considered even in the setting of metastatic disease especially if cancer threatens vital neck structures. The role of external beam radiation (EBRT) to control neck disease in DTC has not been established; it is unclear whether it improves survival. Still, EBRT may help provide local control. Cytotoxic chemotherapy has been used to treat systemic disease but the efficacy is poor. Doxorubicin is FDA-approved for treatment of thyroid cancer, but response rates are low and short-lived. Combination chemotherapy with doxorubicin and agents such as cisplatin have been associated with increased toxicity without improved response or clear impact on survival. ${ }^{21}$

In recent years, new targeted agents for the treatment of advanced thyroid cancer have emerged. The rationale for these agents is that they target and block known aberrancies in thyroid carcinoma, namely constitutive activation of the MAPK and/or PI3 pathway and vascular endothelial growth factor receptors (VEGFRs) (Figure 1). Constitutive activation of the RET receptor via RET/PTC rearrangements and activating mutations of $B R A F$ are commonly reported in PTCs whereas RAS mutations are common in FTC. VEGF, a stimulator of angiogenesis, likely contributes to tumor progression. Importantly, tumors with $B R A F$ mutations are have been associated with increased risk of recurrence. ${ }^{22}$

To date, there have been several Phase II studies evaluating targeted agents in advanced thyroid cancer (Table 1). Most of these agents are tyrosine kinase inhibitors (TKIs) that have activity against pathways that are implicated in DTC. Several agents have multiple targets; some studies include all subtypes of thyroid cancer.

Axitinib and motesanib diphosphate are two oral investigational agents with activity against VEGFRs. A Phase II study of axitinib in advanced thyroid cancer enrolled 60 patients (30 PTC, 15 FTC, 12 MTC, 2 ATC, 1 other) who failed standard therapy. ${ }^{23}$ Documentation of progressive disease was not required. On an intention-to-treat analysis, $30 \%$ of patients (7 PTC, 7 FTC, 3 MTC, 1 ATC) had a partial response and $38 \%$ of patients had stable disease greater than 16 weeks. A Phase II study of motesanib included 93 patients with progressive DTC with evidence of progression. ${ }^{24}$ Of 92 patients available for evaluation, $14 \%$ had a partial response by RECIST and 35\% had durable stable disease for greater than 24 weeks. Common adverse events with VEGFR inhibitors included hypertension, fatigue and diarrhea. 


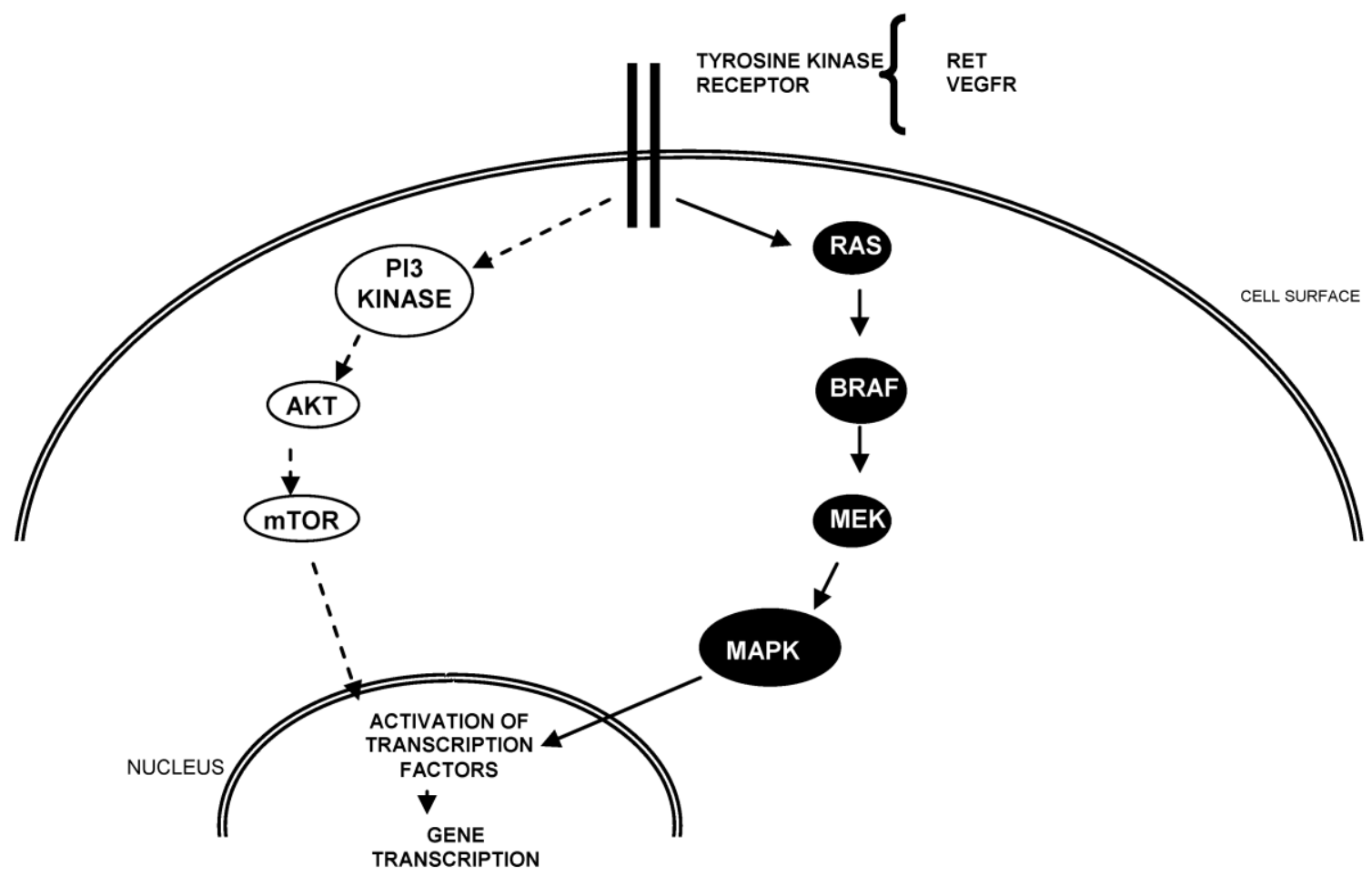

FIGURE I. Schema of key pathways in the development and progression of thyroid cancer.

TABLE I. Targeted agents under clinical evaluation for the treatment of advanced thyroid cancer.

\begin{tabular}{lll}
\hline Compound & Class & Thyroid cancers \\
\hline Axitinib & Tyrosine Kinase Inhibitor (TKI) & DTC, MTC, ATC \\
Motesanib Diphosphate & TKI & DTC \\
Pazopanib & TKI & DTC \\
Sorafenib & TKI & DTC \\
Sunitinib & TKI & DTC, MTC, ATC \\
Thalidomide & Inhibitor of angiogenesis & DTC, MTC \\
Lenalidomide & Inhibitor of angiogenesis & DTC \\
\hline
\end{tabular}

Pazopanib is a TKI targeting several kinases including VEGFR, platelet-derived growth factor, and c-KIT. A Phase II study recruited 39 patients with iodine-refractory DTC with evidence of progression within 6 months of enrollment. ${ }^{25}$ Confirmed partial responses were recorded in $49 \%$ of 37 evaluable patients, the highest response rate yet reported in patients with DTC. The likelihood of response lasting longer than 1 year was calculated at $66 \%$. Common adverse events included fatigue, skin and hair hypopigmentation, diarrhea, and nausea.

Sorafenib is an oral TKI with activity against multiple kinases including BRAF, VEGFR and RET. It is currently indicated for the treatment of advanced renal cell carcinoma and unresectable hepatocellular carcinoma. Sorafenib has shown cytostatic effects in thyroid tumor cells lines, both with and without the presence of BRAF mutations. ${ }^{26,} 27$ Data from several clinical studies of sorafenib for the treatment of advanced thyroid cancer have been reported. One trial evaluated sorafenib in 41 patients with PTC. ${ }^{28} \mathrm{~A}$ total of 6 patients (15\%) had a partial response. Another $56 \%$ of patients had stable disease longer than 6 months. A second study enrolled 55 patients (25 PTC, 19 FTC/HCC, 4 MTC, 5 ATC) with metastatic, iodine-refractory thyroid cancer. ${ }^{29}$ Median PFS was 84 weeks. Sixteen patients had completed BRAF genotyping. Interesting, patients with PTC tumors harboring BRAFV600E mutations had significantly longer PFS (84+weeks) compared to patients with PTC/FTC 
wild type BRAF (54 weeks). Preliminary data from a third Phase II study of sorafenib in advanced thyroid cancer have been presented. ${ }^{30}$ Some 18 patients (10 MTC, 8 DTC) with progressive thyroid cancer were enrolled. Of 10 patients evaluable at 3 months, 9 had stable disease and 1 had a partial response.

Sunitinib is an oral TKI with multiple targets including PDGFR, VEGFR, Kit and RET. Sunitinib is currently indicated for the treatment of metastatic renal cell cancer and for imatinib-resistant gastrointestinal stromal tumors. Preliminary results of three Phase II studies of sunitinib in advanced thyroid cancer have been presented. In one study, 43 subjects (37 DTC, 6 MTC) with progressive disease within the previous 6 months who failed standard therapy were enrolled. ${ }^{31}$ Of 31 patients with DTC who completed 2 cycles, $13 \%$ had a partial response and $68 \%$ had stable disease. In a second trial, 17 patients with advanced thyroid cancer (8 PTC, 4 MTC, 1 ATC, 4 other) were enrolled. Of 15 patients evaluable at 3 months, 1 had a partial response and 12 patients had stable disease. ${ }^{32}$ A third trial of sunitinib in advanced thyroid cancer enrolled 18 patients (15 DTC, 3 MTC). ${ }^{33}$ Although $44 \%$ of patients had a radiographic response on FDG-PET scanning, response rates by RECIST have not been reported.

Promising data on the activity of sorafenib and sunitinib in advanced thyroid cancer have led to the inclusion of both drugs on the NCCN Drug and Biologic Compendium ${ }^{\mathrm{TM}}$. The NCCN Clinical Practice Guidelines in Oncology ${ }^{\mathrm{TM}}$ for Thyroid Carcinoma recommends the use of sorafenib or sunitinib for systemic metastatic disease when a clinical trial is not available or appropriate. ${ }^{34}$

Thalidomide is different than the novel agents described above in that it is not a TKI. Although thalidomide was developed as a sedative in the 1950s, it was found to be teratogenic. However, thalidomide was observed to have antiangiogenic properties, although its mechanism of action is uncertain. A Phase II study of thalidomide enrolled 36 patients (13 PTC, 4 FTC, 8 HCC, 4 insular, 7 MTC) with radioiodine-unresponsive progressive disease. ${ }^{35}$ Of 28 patients available for evaluation, 5 had partial responses and 9 had stable disease. Serious adverse events included infection, pericardial effusion, and pulmonary embolus. More recently, preliminary results from a Phase II study with lenalidomide, a derivative of thalidomide with less toxicity, have been reported. ${ }^{36} \mathrm{~A}$ total of 25 patients with iodine-refractory DTC were initiated on $25 \mathrm{mg}$ of oral lenalidomide daily. Of 18 evaluable patients, $22 \%$ had a partial response and $44 \%$ had stable disease.

Targeted therapy presents new therapeutic op- tions for patients with advanced thyroid cancer. However, criteria for ideal candidates for these new therapies continue to evolve. While early trials included patients with measurable but stable disease, most recent studies have focused on patients with progressive disease as these patients suffer higher morbidity and mortality from their cancers and the toxicities of targeted agents are perhaps better justified. Importantly, randomized-controlled studies are now underway to assess whether targeted agents improve survival.

\section{Pharmacoeconomics}

The cost associated with the increasing incidence of thyroid cancer is not well-understood. A thorough investigation of the literature did not identify published data regarding overall costs related to non-metastatic thyroid cancer, long term management of low-risk thyroid cancer or repeat RAI for refractory disease. Because most thyroid cancer is non-metastatic and the number of these cases continues to increase, the cost related to therapy will surely rise. There has been cost analysis on the use of rhTSH for remnant ablation compared to thyroid hormone withdrawal. ${ }^{37-39}$ A recent study suggests cost effectiveness of rhTSH in the US depends on variations in cost of rhTSH, rates of remnant ablation, productivity loss from time off work and quality of life. ${ }^{37}$

Health care costs of newly metastatic thyroid cancer have been investigated. A retrospective longitudinal cohort study using a large ( 14 million covered lives) US health-insurance claims database, analyzed the costs of health related interventions in 183 patients with newly metastatic thyroid cancer between 2003 and 2005. ${ }^{40}$ Inpatient care was the main driver of the total healthcare expenditure, and represented $43 \%$ of all costs. Radiation therapy was used in $23 \%,{ }^{131}$ I therapy in $19 \%$, thyroid surgery in $13 \%$, and chemotherapy in $11 \%$ of patients. The costs were substantial and totaled $\$ 60,196$ per patient during the first year and \$35,189 during the second year of follow-up. This study did not consider newer targeted agents, which will likely increase costs as well.

Because the use of targeted agents in advanced thyroid cancer is recent, no economic analyses in this setting have been performed. However, pharmacoeconomic studies of sorafenib and similar agents have been published in renal cell carcinoma (RCC) and hepatocellular carcinoma. The National Institute for Health and Clinical Excellence (NICE) in the UK, has issued its final appraisal on the cost-effectiveness of sorafenib for RCC patients in who failed immunotherapy.41 With a value of $£ 65,900$ per quality-adjusted life year (QALY) gained, NICE considered 
sorafenib not to be cost-effective. Similarly, the NICE Committee considered that the incremental cost-effectiveness ratio (ICER) of sorafenib as second-line treatment for RCC patients in whom non-immunotherapy first-line treatment has failed, and who are unsuitable for immunotherapy, would also not be a cost-effective use of the UK National Health Service resources. ${ }^{41}$

The appraisal of sorafenib in hepatocellular carcinoma has also been evaluated. The manufacturer (Bayer) submitted evidence to the NICE Committee in which sorafenib compared with best supportive care (BSC) produced a base-case ICER of $£ 64,754$ per QALY gained. The NICE Committee, however, issued a preliminary appraisal document with an ICER of $£ 76,067$, and stated that sorafenib as a treatment for advanced hepatocellular carcinoma in patients for whom surgical or locoregional therapies had failed or were not suitable, is not cost-effective. ${ }^{42}$ From the U.S. payer perspective, a recent economic evaluation of sorafenib in unresectable hepatocellular carcinoma versus BSC estimated that the ICER was $\$ 62,473$ per QALY gained. ${ }^{43}$ Of note, these studies were performed in malignancies in which the efficacy of sorafenib has been demonstrated in randomized-controlled studies. Nevertheless, these evaluations do illustrate the complex issue of cost and effectiveness with new targeted agents.

The addition of targeted drugs has the potential to substantially alter the cost of treatment of advanced thyroid cancer. Thus, as resources are scarce, pharmacoeconomic and quality of life analyses of these different drugs in the US setting will be mandatory.

\section{Conclusions}

The cost associated with the management of thyroid cancer is bound to increase in coming years. Part of this increase will likely be from costs incurred for the standard treatment of PTC, as this subtype of thyroid cancer has seen a large increase in diagnosis. Controlling cost while still providing optimal care will require better risk stratification in low-risk patients. While certain factors such as increasing age or male gender increase a patient's chance of having progressive disease, current diagnostic tools are inadequate to accurately predict which patients will do well with minimal therapy. While surgery remains necessary for adequate staging, more judicious use of RAI may help control costs. In the future, newer tools, such as molecular genetics to detect more or less aggressive cancers, may aid clinicians in recommending the most appropriate therapy.

Although patients with advanced thyroid cancer represent a minority of patients, they require contin- ued evaluation and therapy which will also increase health care expenditures. Although many of these patients receive targeted therapy as part of clinical trials, the availability of sorafenib and sunitinib provides options when trials are not available. These agents do have significant toxicities and costs; therefore, identifying which patients with metastatic disease are likely to benefit novel therapies remains an important research question. At present, results from randomized studies are needed to investigate whether targeted agents improve survival. Whether these agents are cost effective remains an unknown but important question.

\section{Conflict of Interest}

The authors have declared that no conflict of interest exists.

\section{References}

1. [Internet] American Cancer Society. http://www.cancer.org.

2. [Internet] Waguespack S WS, Ross J, Bleyer A. National Cancer Institute AYA Monograph Thyroid Cancer. www.seer.cancer.gov/publications/aya/12_thyroid.pdf.

3. Chen AY, Jemal A, Ward EM. Increasing incidence of differentiated thyroid cancer in the United States, 1988-2005. Cancer 2009;115(16):3801-7.

4. Amdur RJ. Incidence, Prevalence, Recurrence, and Mortality of Differentiated Thyroid Cancer. In: Essentials of Thyroid Cancer Management. US: Springer; 2005.

5. Dean DS, Gharib H. Epidemiology of thyroid nodules. Best practice \& research 2008;22(6):901-11.

6. Kovacs GL, Gonda G, Vadasz G, et al. Epidemiology of thyroid microcarcinoma found in autopsy series conducted in areas of different iodine intake. Thyroid 2005;15(2):152-7.

7. Harach HR, Franssila KO, Wasenius VM. Occult papillary carcinoma of the thyroid. A "normal" finding in Finland. A systematic autopsy study. Cancer 1985;56(3):531-8.

8. Fukunaga FH, Yatani R. Geographic pathology of occult thyroid carcinomas. Cancer 1975;36(3):1095-9.

9. Mazzaferri EL, Jhiang SM. Long-term impact of initial surgical and medical therapy on papillary and follicular thyroid cancer. Am J Med 1994;97(5):418-28.

10. Hay ID, Bergstralh EJ, Goellner JR, Ebersold JR, Grant CS. Predicting outcome in papillary thyroid carcinoma: development of a reliable prognostic scoring system in a cohort of 1779 patients surgically treated at one institution during 1940 through 1989. Surgery 1993;114(6):1050-7.

11. DeGroot LJ, Kaplan EL, Straus FH, Shukla MS. Does the method of management of papillary thyroid carcinoma make a difference in outcome? World J Surg 1994;18(1):123-30.

12. Mazzaferri EL, Young RL. Papillary thyroid carcinoma: a 10 year follow-up report of the impact of therapy in 576 patients. Am J Med 1981;70(3):511-8.

13. Sosa JA, Udelsman R. Total thyroidectomy for differentiated thyroid cancer. J Surg Oncol 2006;94(8):701-7.

14. Mazzaferri EL. Thyroid remnant 131I ablation for papillary and follicular thyroid carcinoma. Thyroid 1997;7(2):265-71.

15. DeGroot LJ, Kaplan EL, McCormick M, Straus FH. Natural history, treatment, and course of papillary thyroid carcinoma. J Clin Endocrinol Metab 1990;71(2):414-24.

16. Hay ID. Papillary thyroid carcinoma. Endocrinol Metab Clin North Am 1990;19(3):545-76. 
17. Hay ID, Grant CS, van Heerden JA, et al. Papillary thyroid microcarcinoma: a study of 535 cases observed in a 50 -year period. Surgery 1992;112(6):1139-46.

18. Hay ID, Thompson GB, Grant CS, et al. Papillary thyroid carcinoma managed at the Mayo Clinic during six decades (1940-1999): temporal trends in initial therapy and long-term outcome in 2444 consecutively treated patients. World J Surg 2002;26(8):879-85.

19. Vaisman A, Orlov S, Yip J, et al. Application of post-surgical stimulated thyroglobulin for radioiodine remnant ablation selection in low-risk papillary thyroid carcinoma. Head \& Neck 2010;32(6):689-98.

20. Biondi B, Filetti S, Schlumberger M. Thyroid-hormone therapy and thyroid cancer: a reassessment. Nat Clin Pract Endocrinol Metab 2005;1(1):32-40.

21. Lessin L. Chemotherapy for Differentiated Papillary or Follicular Thyroid Cancer. Totowa, NJ: Humana Press; 2006.

22. Costa AM, Herrero A, Fresno MF, et al. BRAF mutation associated with other genetic events identifies a subset of aggressive papillary thyroid carcinoma. Clin Endocrinol (Oxf) 2008;68(4):618-34.

23. Cohen EE, Rosen LS, Vokes EE, et al. Axitinib Is an Active Treatment for All Histologic Subtypes of Advanced Thyroid Cancer: Results From a Phase II Study. J Clin Oncol 2008;26(29):4708-13.

24. Sherman SI, Wirth LJ, Droz JP, et al. Motesanib diphosphate in progressive differentiated thyroid cancer. $\mathrm{N}$ Engl J Med 2008;359(1):31-42.

25. Bible KC, Suman VJ, Molina JR, et al. Efficacy of pazopanib in progressive, radioiodine-refractory, metastatic differentiated thyroid cancers: results of a phase 2 consortium study. Lancet Oncol; 2010;11(10):962-72.

26. Salvatore G, De Falco V, Salerno P, et al. BRAF is a therapeutic target in aggressive thyroid carcinoma. Clin Cancer Res 2006;12(5):1623-9.

27. Kim S, Yazici YD, Calzada G, et al. Sorafenib inhibits the angiogenesis and growth of orthotopic anaplastic thyroid carcinoma xenografts in nude mice. Molecular cancer therapeutics 2007;6(6):1785-92.

28. Kloos RT, Ringel MD, Knopp MV, et al. Phase II trial of sorafenib in metastatic thyroid cancer. J Clin Oncol 2009;27(10):1675-84.

29. Brose MS TA, Redlinger M, Harlacker $K$, Redlinger $C$, Chalian AA, Flaherty KT, Loevner LA, Mandel SJ, O'Dwyer PJ. Effect of BRAFV600E on response to sorafenib in advanced thyroid cancer patients. J Clin Oncol 2009;27:15s.

30. Ahmed M BY, Riddell AM, Whittaker S, Newbold K, Harrington $\mathrm{K}$, Marais R, Nutting CM. Preliminary results of an open labelled phase 2 study evaluating the safety and efficacy of sorafenib in metastatic advanced thyroid cancer. J Clin Oncol 2008;26: a6060.

31. Cohen EE NB, Cullen KJ, Wong SJ, Wade JL, Ivy SP, Villaflor VM, Seiwert TY, Nichols K, Vokes EE. Phase 2 study of sunitinib in refractory thyroid cancer. J Clin Oncol 2008;26: a6025.

32. Ravaud A dlFC, Courbon F, Asselineau J, Klein M, Nicoli-Sire P, Bournaud C, Delord J, Weryha G, Catargi B. Sunitinib in patients with refractory advanced thyroid cancer: the THYSU phase II trial. J Clin Oncol 2008;26: 6058.

33. Goulart B CL, Martins RG, Eaton K, Kell E, Wallace S, Capell P, Mankoff D. Phase II study of sunitinib in iodine refractory, well-differentiated thyroid cancer (WDTC) and metastatic medullary thyroid carcinoma (MTC). J Clin Oncol 2008;26: a6062.

34. [Internet] National Comprehensive Cancer Network. Thyroid Carcinoma. physician_gls/PDF/thyroid.pdf.
35. Ain KB, Lee C, Williams KD. Phase II trial of thalidomide for therapy of radioiodine-unresponsive and rapidly progressive thyroid carcinomas. Thyroid 2007;17(7):663-70.

36. Ain K LC, Holbrook KM, Dziba JM, Williams KD. Phase II study of lenalidomide in distantly metastatic, rapidly progressive, and radioiodine-unresponsive thyroid carcinomas: preliminary results. J Clin Oncol 2008;26:a6027.

37. Wang TS, Cheung K, Mehta P, et al. To Stimulate or Withdraw? A Cost-Utility Analysis of Recombinant Human Thyrotropin Versus Thyroxine Withdrawal for Radioiodine Ablation in Patients with Low-Risk Differentiated Thyroid Cancer in the United States. J Clin Endocrinol Metab 2010 Apr;95(4):1672-80.

38. Mernagh P, Suebwongpat A, Silverberg J, Weston A. Cost-Effectiveness of Using Recombinant Human Thyroid-Stimulating Hormone before Radioiodine Ablation for Thyroid Cancer: The Canadian Perspective. Value Health 2010;13(2):180-7.

39. Mernagh P, Campbell S, Dietlein M, et al. Cost-effectiveness of using recombinant human TSH prior to radioiodine ablation for thyroid cancer, compared with treating patients in a hypothyroid state: the German perspective. Eur J Endocrinol 2006;155(3):405-14.

40. Berger A EJ, Chung K, Ngyuyen A, Stepan D, Oster G. Healthcare (HC) utilization and costs in patients (pts) with newly diagnosed metastatic thyroid cancer (mTC). J Clin Oncol 2007;25:17082.

41. [Internet] NICE. NICE technology appraisal guidance 178 Bevacizumab (first-line), sorafenib (first- and second-line), sunitinib (second-line) and temsirolimus (first-line) for the treatment of advanced and/or metastatic renal cell carcinoma. www.nice.org.uk/TA178.

42. [Internet] NICE. NICE technology appraisal guidance 189 Sorafenib for the treatment of advanced hepatocellular carcinoma. http://guidance.nice.org.uk/TA189.

43. Carr BI, Carroll S, Muszbek N, Gondek K. Economic evaluation of sorafenib in unresectable hepatocellular carcinoma. Journal of gastroenterology and hepatology 2010; 25(11):1739-46. 